\title{
Improvement of long-term risks of cardiovascular events associated with community-based disease management in Chinese patients of the Xinjiang autonomous region of China
}

Yang Li ${ }^{1}$, Cai Minzhang ${ }^{1}$, Ma Minghui ${ }^{2}$, Huang Xinmiao ${ }^{2}$, Liu Laixin ${ }^{3}$, Wang Bei ${ }^{4}$, Zhu Weihai ${ }^{2}$, Zhe Wei ${ }^{3}$, Guan Yumei ${ }^{5}$, Thitima Kongnakorn ${ }^{6}$, Ying Xiao ${ }^{6}$, Siyang Peng ${ }^{6}$, David Hughes ${ }^{7}$, Naranjargal Dashdorj ${ }^{8}$ and Thomas Hach ${ }^{*}$

\begin{abstract}
Background: A recent community-based disease management (CBDM) pilot study reported a 20.5\% prevalence of hypertension and a 0.5 and 3.6\% prevalence of stroke and coronary heart disease (CHD), respectively, in an elderly population (mean age 65 years) in the Xin Jiang autonomous region of China. The CBDM was initiated in 2013 as an essential public health service; however, the potential long-term impact of CBDM on cardiovascular (CV: CHD and stroke) events is unknown. The objective of the study was to understand the long-term impact of CBDM interventions on CV risk factors using disease-model simulation based on a single-arm experimental study.

Methods: A discrete event simulation was developed to evaluate the impact of CBDM on the long-term CV risk among patients with hypertension, in China's Xin Jiang autonomous region. The model generated pairs of identical patients; one receives CBDM and one does not (control group). Their clinical courses were simulated based on time to $\mathrm{CV}$ events (CHD and strokes), which are estimated using published risk equations. The impact of CBDM was incorporated as improvement in systolic blood pressure (SBP) based on observations from the CBDM study. The simulation estimated the number of $\mathrm{CV}$ events over patients' lifetimes.

Results: During a 2-year follow up, the CBDM led to an average reduction of $8.73 \mathrm{mmHg}$ in SBP from baseline, and a $42 \%$ reduction in smoking. The discrete event simulation showed that, in the control group, the model estimated incidence rates of 276, 1789, and 616 per 100,000 individuals for lifetime CHD, stroke, and CV-related death, respectively. The impact of CBDM on SBP translated into reductions of 8, 28, and 23\% in CHD, stroke, and CV-related deaths, respectively. Taking into account CBDM's reduction of both SBP and smoking, deaths from CHD, stroke, and CV-related deaths were reduced by 12,30 , and $26 \%$, respectively.

(Continued on next page)
\end{abstract}

\footnotetext{
* Correspondence: thomas.hach@novartis.com

${ }^{9}$ Novartis Pharma AG, Fabrikstrasse 12-1.03.14A, 4056 Basel, Switzerland

Full list of author information is available at the end of the article
}

C C The Author(s). 2020 Open Access This article is licensed under a Creative Commons Attribution 4.0 International License, which permits use, sharing, adaptation, distribution and reproduction in any medium or format, as long as you give appropriate credit to the original author(s) and the source, provide a link to the Creative Commons licence, and indicate if changes were made. The images or other third party material in this article are included in the article's Creative Commons licence, unless indicated otherwise in a credit line to the material. If material is not included in the article's Creative Commons licence and your intended use is not permitted by statutory regulation or exceeds the permitted use, you will need to obtain permission directly from the copyright holder. To view a copy of this licence, visit http://creativecommons.org/licenses/by/4.0/. The Creative Commons Public Domain Dedication waiver (http://creativecommons.org/publicdomain/zero/1.0/) applies to the data made available in this article, unless otherwise stated in a credit line to the data. 
(Continued from previous page)

Conclusions: The implementation of CBDM in China's Xinjiang autonomous region is expected to significantly reduce incidences of $\mathrm{CHD}$, strokes, and CV-related deaths.

Keywords: Congestive heart failure (CHD), Cardiovascular disease (CVD), Community-based disease management (CBDM), Discrete-event simulation (DES) model, Hypertension, Stroke, Myocardial infarction (MI)

\section{Background}

Cardiovascular diseases (CVD) are the leading causes of death for patients in both urban and rural areas of China. Currently, $44.6 \%$ of deaths in rural areas and $42.5 \%$ in urban areas are caused by CVDs. Hypertension is the primary risk factor for stroke and coronary heart disease (CHD). According to a national survey in China in 2012, the prevalence of hypertension in adults aged 18 years or older was $25.2 \%$. It is estimated that 270 million Chinese patients are hypertensive and more than half of CVDs are associated with hypertension in China [1].

Awareness, treatment, and control rates of hypertension are still relatively low in China, at 26.1, 22.8, and $6.1 \%$, respectively. Overestimated blood pressure (BP) control rates may contribute to therapeutic inertia and poor BP control among treated patients with hypertension $[2,3]$.

\section{Community based disease management}

A community-based disease management (CBDM) pilot study was conducted to analyze the effect of follow-up management for chronic disease on community hypertension management in the Xinjiang Uygur Autonomous Region in China. The CBDM study reported a $20.5 \%$ prevalence of hypertension and 0.5 and $3.6 \%$ prevalence of stroke and CHD, respectively, in an elderly population (mean age 65 years). The CBDM was initiated in 2013 as an essential public health service; however, the potential long-term impact of CBDM on cardiovascular (CV) events is unknown.

The objective of the present study was to understand the long-term impact of CBDM interventions on $\mathrm{CV}$ risk factors and events using a simulation model, and to use disease-model simulation forecasts to predict how various interventions would affect $\mathrm{CV}$ outcomes such as stroke, CHD, and mortality. Risk factors considered included control of blood pressure, lipids, and smoking.

\section{Methods}

\section{Community based disease management study}

The CBDM study was initiated in 2013 in Xinjiang Uygur Autonomous Region. Of the 21.81 million local residents, 2800 patients with hypertension from 39 community and township hospitals were recruited with a stratified multistage random sampling method. Patients under the basic public health services management conforming to the following conditions were enrolled: (a) age $\geq 35$ years, (b) suffering from primary hypertension, and (c) participating in this management program of hypertensive patients, which acts as a basic public health service. This was a single-arm experimental study; all patients received interventions for BP, which included medication, life-style management, dietary recommendations, and exercise. Risk factors that could influence the outcome of hypertension control were captured at baseline and at the end of the two-year follow-up, including but not limited to, age, gender, BP, smoking status, diabetes status, and history of CVD. The CBDM population and the efficacy of the intervention such as systolic BP reduction and smoking reduction were used to inform the simulation model.

The study was approved by the ethics committees of the Peking University, School of Public Health, and the study was conducted in accordance with the International Council on Harmonization (ICH, E6) guidelines and Declaration of Helsinki and its subsequent revisions. The data was collected by the Xinjiang CDC and the written patient consents were received prior to the survey.

\section{Discrete-event simulation model}

A discrete-event simulation (DES) model was developed in Microsoft Excel to evaluate the impact of CBDM on long-term $\mathrm{CV}$ (CHD and stroke) risks among patients with hypertension. The DES approach for disease modeling is a well-established technique that is in agreement with the modeling good research practices recommended by the International Society for Pharmacoeconomics and Outcomes Research (ISPOR), and is widely used in published models [4-6]. The model was designed to simulate clinical events associated with high $\mathrm{BP}$, including acute myocardial infarction (MI), coronary death, stroke, stroke-related death, and background mortality over a 30 -year time horizon. DES was selected because of the dynamic nature of events and unpredictable timing of disease- and treatment-related events. This approach used time-to-event calculations to simulate the clinical course of these patients. The core of this model was a set of equations representing the physiological pathways pertinent to diseases and their complications. Depending on the baseline characteristics, each patient followed a distinct disease pathway. The event rate and life-year 
outcome for the overall modeled cohort and longitudinal event outcomes were reported.

At the beginning of each simulation, individual patients were created by sampling from patient profiles of those who participated in the CBDM study. Each patient profile included age, gender, systolic blood pressure (SBP), total cholesterol, high-density lipoprotein (HDL), diabetes status, smoking status, and body mass index (BMI). In addition, the disease history of stroke, CVD, and CHD were also assigned to each patient at baseline. After the patients were created, they were "cloned" to produce two identical cohorts. This ensured that the comparisons were not affected by differences in baseline characteristics. One patient in each pair was assumed to experience changes in $\mathrm{CV}$ (CHD and stroke) risk factors due to CBDM intervention (including changes in SBP, lipid levels, and smoking cessation), while the other patient was assumed to receive no intervention. The impact of intervention was assumed to take place within the first 3 months after entering the model and to last for the entire model time horizon. Any exacerbation of the baseline condition was not considered. Patients receiving no intervention were assumed to have no change in these risk factors, except for age, throughout the model time horizon. Age and gender-specific background mortality was considered to account for the health deterioration of a general population.

Each patient was exposed to various risks: CHD (acute MI and coronary death), stroke, and background death, which were handled as competing risks. The competing risks were implemented by using a series of risk equations to translate the patient's risk factors (e.g. age, gender, and SBP) into a distribution of failure times that were specific to the individual. A time for each event can then be assigned to each individual by sampling from the corresponding distribution. The prediction equations for CHD (i.e. acute MI and coronary death) were obtained from the Chinese Multi-Provincial Cohort Study (CMCS), with risk factors including age, BP, total cholesterol, HDL, diabetes, and smoking (Table 1) [7]. The CMCS was conducted in 30,121 Chinese adults aged from 35 to 64 with a follow-up of 10 years (1992 to 2002) for CHD events. The CMCS cohort had 191 hard CHD events (including coronary death and MI) and 625 total deaths.

The prediction equation for stroke was obtained from the People's Republic of China - United States of America (PRC-USA) study with risk factors including age, BP, BMI, total cholesterol, smoking, and diabetes (Table 2) [8]. PRC-USA collaborative study was conducted in 9903 participants with 17 years of follow-up until the year of 2000; 266 strokes and 105 CHD events occurred.

Twenty-eight day case fatality rates for $\mathrm{CHD}$ and stroke were estimated based on the Sino-MONICA
Table 1 Variables of CHD risk equation used in model base case

\begin{tabular}{|c|c|c|}
\hline & Male & Female \\
\hline & Coefficient & Coefficient \\
\hline Age & 0.07 & 0.07 \\
\hline SBP - optimal & -0.51 & -0.5 \\
\hline SBP - normal & Reference & Reference \\
\hline SBP - high normal & 0.21 & -0.87 \\
\hline SBP - stage 1 hypertension & 0.33 & 0.34 \\
\hline SBP - stage 2-4 hypertension & 0.77 & 0.47 \\
\hline Total cholesterol $<160 \mathrm{mg} / \mathrm{dL}$ & -0.51 & 0.18 \\
\hline Total cholesterol $160-199 \mathrm{mg} / \mathrm{dL}$ & Reference & Reference \\
\hline Total cholesterol $200-239 \mathrm{mg} / \mathrm{dL}$ & 0.07 & 0.13 \\
\hline Total cholesterol $240-279 \mathrm{mg} / \mathrm{dL}$ & 0.32 & 0.14 \\
\hline Total cholesterol $\geq 280 \mathrm{mg} / \mathrm{dL}$ & 0.52 & 1.67 \\
\hline $\mathrm{HDL}-\mathrm{C}<35 \mathrm{mg} / \mathrm{dL}$ & -0.25 & 0.62 \\
\hline HDL-C 35-44 mg/dL & 0.01 & 0.3 \\
\hline HDL-C $45-49$ mg/dL & Reference & 0.08 \\
\hline HDL-C 50-59 mg/dL & -0.07 & Reference \\
\hline $\mathrm{HDL}-\mathrm{C} \geq 60 \mathrm{mg} / \mathrm{dL}$ & -0.4 & -0.78 \\
\hline Diabetes (yes $=1$, no $=0$ ) & 0.09 & 0.18 \\
\hline Smoking (yes = 1, no $=0$ ) & 0.62 & -0.95 \\
\hline Survival & 0.9895 & 0.9961 \\
\hline
\end{tabular}

$C H D$ coronary heart disease, $H D L-C$ high-density lipoprotein cholesterol, SBP systolic blood pressure

SBP optimal: $\leq 120 \mathrm{mmHg}$; SBP normal $120-130 \mathrm{mmHg}$; SBP high normal $130-$ $140 \mathrm{mmHg}$; SBP stage 1 hypertension $140-160 \mathrm{mmHg}$; SBP stage 2-4 hypertension $\geq 160 \mathrm{mmHg}$

Table 2 Variables of stroke risk equation used in model base case

\begin{tabular}{lll}
\hline & $\begin{array}{l}\text { Male } \\
\text { Coefficient }\end{array}$ & $\begin{array}{l}\text { Female } \\
\text { Coefficient }\end{array}$ \\
\hline Age & 0.07 & 0.09 \\
SBP $<\mathbf{1 2 0}$ & -0.55 & -0.83 \\
SBP 120-129 & Reference & Reference \\
SBP $\mathbf{1 3 0 - 1 3 9}$ & 0.40 & 0.23 \\
SBP $\mathbf{1 4 0 - 1 5 9}$ & 0.81 & 0.80 \\
SBP $\mathbf{1 6 0 - 1 7 9}$ & 1.70 & 1.37 \\
SBP $\geq \mathbf{1 8 0}$ & 2.53 & 1.85 \\
BMI $<\mathbf{2 4}$ & Reference & Reference \\
BMI $\geq \mathbf{2 4}$ & 0.29 & 0.68 \\
Total cholesterol $<\mathbf{3 . 6 2} \mathbf{~ m m o l / L}$ & Reference & Reference \\
Total cholesterol $\mathbf{3 . 6 2 - 5 . 1 6 ~} \mathbf{~ m m o l / L}$ & -0.01 & -0.09 \\
Total cholesterol $\geq \mathbf{5 . 1 7} \mathbf{~ m m o l / L}$ & 0.30 & 0.27 \\
Smoking & 0.71 & 0.47 \\
Diabetes & 0.07 & 0.96 \\
Survival & 0.9835 & 0.9948 \\
Survival & 0.9895 & 0.9961 \\
\hline BMI body mass & &
\end{tabular}

$B M I$ body mass index, SBP systolic blood pressure 
study for the Chinese population (Table 3) [9]. Background mortality was obtained from the life table for China $[10,11]$. The time-to-event calculations were estimated at baseline. The event that was estimated to occur first was recorded by the model and triggered the update of patient characteristics and estimation of the time-tonext event. Other than the time that the events occurred, all time-to-event and clinical measurements were updated in the model based on a quarterly time unit for the first 2 years, then based on an annual time unit beyond 2 years until the end of model time horizon. The quarterly time unit was intended to provide granularity that was consistent with the quarterly assessment schedule during the two-year follow-up of the CBDM study.

\section{Study outcomes and analysis}

A cohort of virtual patients that represented the patient population of 2800 from the CBDM study was generated. Each simulated patient was run through the baseline control and intervention scenarios for 30 iterations over a 30-year time horizon.

Three intervention scenarios were explored in the model, including 1) SBP reduction only, 2) SBP reduction and smoking cessation, and 3) SBP reduction, smoking cessation, and lipid control. Clinical inputs of SBP reduction and smoking cessation were obtained from the clinical results of the CBDM study. Lab values for lipid status were not available from the CBDM study. Therefore, based on CBDM physician suggestion, a $10 \%$ increase in HDL and a $10 \%$ decrease in total cholesterol from the baseline was assumed for lipid control. The following health outcomes generated from the DES model were (1) total event count and event rate of CHD, (2) total event count and event rate of stroke, (3) mortality due to CHD, (4) mortality due to stroke and (5) total death.

Subgroup analysis was also conducted for patients aged $<60$ years versus patients aged $\geq 60$ years, male versus female, and patients with SBP $<140 \mathrm{mmHg}$ versus patients with SBP $\geq 140 \mathrm{mmHg}$.

Table 3 Case fatality rate for CHD and stroke, by gender and age group

\begin{tabular}{llllll}
\hline & \multicolumn{2}{l}{ CHD } & & & \multicolumn{2}{l}{ Stroke } \\
\cline { 2 - 3 } & Male & Female & & Male & Female \\
\hline $\mathbf{3 5 - 4 4}$ years, \% & 30 & 50 & & 6 \\
$\mathbf{4 5 - 5 4}$ years, \% & 38 & 42 & & 11 & 10 \\
$\mathbf{5 5 - 6 4}$ years, \% & 41 & 43 & 17 & 15 \\
$\mathbf{6 5 - 7 4}$ years, \% & 45 & 51 & & 27 & 25 \\
$\mathbf{7 5 - 8 4}$ years, \% & 53 & 57 & & 31 & 28 \\
\hline
\end{tabular}

CHD coronary heart disease

\section{Results}

Clinical outcomes of CBDM study

Baseline population characteristics of the Chinese population from the CBDM study that were simulated in the DES model are reported in Table 4. The characteristics were well balanced between male and female, except for smoking status. Smoking was more prevalent in males (22\%) than in females (1\%).

For the overall population, the intervention program in the CBDM study was found to be associated with a reduction of $8.73 \mathrm{mmHg}$ in SBP and $42 \%$ reduction of smoking. Table 5 presents the SBP reduction by subgroups from the CBDM study. SBP reduction over the two-year follow-up was higher in females $(9.28 \mathrm{mmHg})$ than in males $(7.94 \mathrm{mmHg})$. SBP reduction between the $<60$ - and $\geq 60$-year age groups were similar. The subgroup with baseline SBP $\geq 140 \mathrm{mmHg}$ was reported to have the highest SBP reduction of $18.28 \mathrm{mmHg}$.

\section{Long-term projection of clinical outcomes in overall population}

The CBDM population with hypertension intervention was found to be associated with significant improvement in simulated 30-year risk across all clinical outcomes. In the overall population, the impact of CBDM on SBP resulted in reductions of $8,28,5$, and $28 \%$ in CHD events, strokes, CHD-related deaths, and stroke-related deaths, respectively. Taking into account CBDM's reduction of both SBP and smoking, CHD, stroke, CHD-related deaths and stroke-related deaths fell by 12, 30, 9, and $30 \%$, respectively. Event rates were further reduced when smoking cessation and lipid control in terms of increased HDL and decreased total cholesterol, were applied (20, 46, 18, and 45\% for CHD, stroke, CHD-related deaths and stroke-related deaths, respectively). The event rate per 100,000 people simulated with the overall population over the 30 -year time horizon is presented in Table 6.

Figures 1 and 2 present the relative risks of clinical events between the three scenarios with interventions versus the one scenario without intervention. The relative risks and their 95\% CI of intervention group versus no intervention group for $\mathrm{CHD}$ and for stroke were lower than one for all intervention scenarios indicating significant risk reduction with the intervention group. Risk reduction was greatest (20 and $46 \%$ reduction in CHD and stroke, respectively) when impact of intervention on SBP, smoking cessation, and lipid profiles were considered.

Figure 3 illustrates the impact of intervention in the CBDM study on mortality due to CHD or stroke over a 30-year time horizon. Compared to the scenario without intervention, the addition of SBP control, smoking 
Table 4 Baseline population characteristics

\begin{tabular}{|c|c|c|c|}
\hline & Full population $(\boldsymbol{N}=2800)$ & Male $(\boldsymbol{N}=1199)$ & Female $(\boldsymbol{N}=1601)$ \\
\hline Age & $65.4(11.1)$ & $65.4(11.4)$ & $65.4(10.8)$ \\
\hline SBP $(\mathrm{mmHg})$, mean (SD) & $144.3(20.0)$ & $143.9(20.1)$ & $144.6(20.0)$ \\
\hline SBP $<120 \mathrm{mmHg}, \%$ & 4 & 4 & 4 \\
\hline SBP $120-129 \mathrm{mmHg}, \%$ & 13 & 13 & 13 \\
\hline SBP $130-139 \mathrm{mmHg}, \%$ & 19 & 20 & 19 \\
\hline SBP $140-159 \mathrm{mmHg}, \%$ & 40 & 40 & 40 \\
\hline SBP $160-179 \mathrm{mmHg}, \%$ & 17 & 18 & 16 \\
\hline $\mathrm{SBP} \geq 180 \mathrm{mmHg}, \%$ & 7 & 7 & 7 \\
\hline Total cholesterol (mg/dL), mean (SD) & $212.3(22.0)$ & $213.3(21.5)$ & $211.5(22.3)$ \\
\hline HDL (mg/dL), mean (SD) & $67.4(6.2)$ & $69.6(11.1)$ & $65.8(5.3)$ \\
\hline LDL (mg/dL), mean (SD) & $117.9(16.5)$ & $117.8(16.2)$ & $118.1(16.6)$ \\
\hline Female, \% & 57 & 0 & 100 \\
\hline Diabetes, \% & 16 & 14 & 19 \\
\hline Smoking, \% & 11 & 22 & 1 \\
\hline
\end{tabular}

$H D L$ high-density lipoprotein, $L D L$ low-density lipoprotein, $S B P$ systolic blood pressure, $S D$ standard deviation

cessation, and lipid control contributed to a reduction of mortality due to CHD or stroke.

\section{Long-term projection of clinical outcomes in subgroups}

Subgroup analysis of long-term projection of clinical outcomes was conducted in the CBDM population aged $<60$ years versus $\geq 60$ years, male versus female, and baseline SBP $<140 \mathrm{mmHg}$ versus baseline $\mathrm{SBP} \geq 140$ mmHg.

The subgroup analysis results showed that the CBDM population with hypertension intervention was associated with improvement in simulated 30-year risk across all clinical outcomes (CHD, stroke, and death related to CHD and stroke) in all subgroups with an exception in the subgroup with baseline SBP below $140 \mathrm{mmHg}$ across all clinical outcomes. The greatest reduction in event rates was seen in the subgroup with $\mathrm{SBP} \geq 140 \mathrm{mmHg}$. Patients aged $<60$ years, male, or SBP $\geq 140 \mathrm{mmHg}$ were reported to benefit more from the hypertension control

Table 5 SBP reduction in subgroups

\begin{tabular}{llll}
\hline Subgroups & $\begin{array}{l}\text { Sample } \\
\text { size }\end{array}$ & \multicolumn{2}{l}{ SBP reduction $(\mathrm{mmHg})$} \\
\cline { 3 - 4 } & 1199 & -7.9436 & Mean \\
\hline Male & 1601 & -9.2856 & 25.3237 \\
Female & 890 & -8.9761 & 26.3045 \\
Age $<\mathbf{6 0}$ & 1910 & -8.4864 & 24.5032 \\
Age $\geq \mathbf{6 0}$ & 1014 & 8.9768 & 15.7911 \\
SBP $<\mathbf{1 4 0} \mathbf{~} \mathbf{m H g}$ & 1786 & -18.2826 & 16.7164 \\
SBP $\geq \mathbf{1 4 0} \mathbf{~} \mathbf{m H g}$ & & &
\end{tabular}

$S B P$ systolic blood pressure, $S D$ standard deviation program over the long term than older patients, females, or low SBP subgroups, respectively. Table 7 presents the event rates per 100,000 people and relative risks of CHD, stroke, CHD-related death, and stroke-related death across all subgroups.

\section{Discussion}

This study aimed to evaluate the potential impact of improved management of hypertension and other known $\mathrm{CV}$ (CHD and stroke) risk factors (dyslipidemia and smoking) in China over a 30-year time horizon. Our analysis showed that BP intervention implemented in the CBDM study, which included medication, life-style management, dietary recommendations, and exercise can lead to a $20 \%$ risk reduction in CHD events and $45 \%$ risk reduction in stroke events. The addition of smoking cessation and dyslipidemia control would further reduce the number of CHD and stroke events. As stroke is generally considered more dangerous than a CHD event this may have led to underreporting of stroke prevalence in the pilot study. Hence, it seems even more important to foster education and preventive measures.

Along with the clarion call for expanding Universal Health Coverage globally [12], the World Health Organization (WHO) has also emphasized the urgency of strengthening primary care services worldwide [13]. In addition, the management of hypertension, one of the most common chronic health conditions and the number one risk factor for overall mortality, has been suggested as a proxy measure of clinical performance [14, 15].

Dyslipidemia and smoking control were assessed as additional interventions to hypertension control. The 
Table 6 Event rate per 100,000 people in overall population

\begin{tabular}{lllll}
\hline Intervention & CHD & Stroke & CHD-related death & Stroke-related death \\
\hline Without intervention & 276.0 & 1788.9 & 139.4 & 476.2 \\
SBP & 253.8 & 1291.0 & 131.6 & 342.8 \\
SBP + smoking cessation & 242.1 & 1255.0 & 125.7 & 332.7 \\
SBP + smoking cessation + lipid control & 218.1 & 960.0 & 112.7 & 259.3 \\
\hline
\end{tabular}

$C H D$ coronary heart disease, SBP systolic blood pressure

further reduction in CV (CHD and stroke) events highlights the importance of a multifactorial approach to target $\mathrm{CV}$ (CHD and stroke) event reduction. The results give a clear indication of the potential gains in mortality and morbidity by addressing three of the common and treatable risk factors.

The CBDM population represented an older and more hypertensive population in the Xinjiang province. An epidemiological survey that enrolled 14,618 adults aged $\geq 35$ years from Xinjiang found that $42 \%$ of the population had SBP $>140 \mathrm{mmHg}$ [16], whereas in the CBDM population, over $60 \%$ of patients had SBP $>140 \mathrm{mmHg}$. In comparison with the general population in China, the CBDM population had higher total cholesterol $(212 \mathrm{mg} / \mathrm{dL}$ vs $174 \mathrm{mg} / \mathrm{dL})$ and higher HDL $(67 \mathrm{mg} / \mathrm{dL}$ vs $46 \mathrm{mg} / \mathrm{dL})$ [17], smoking was less prevalent in the CBDM population than in the general Chinese population $(52.1 \%$ in male and $2.7 \%$ in female) [18]. If CBDM could be applied to the total population of Xinjiang Uygur Autonomous Region (21.8 million), this approach could save approximately 27,000 deaths from CHD or stroke each

SBP/Smoking/Lipid

$0.797(0.760-0.837)$

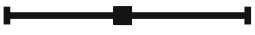

SBP/Smoking

$0.881(0.841-0.924)$

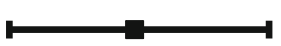

SBP

$0.923(0.881-0.967)$
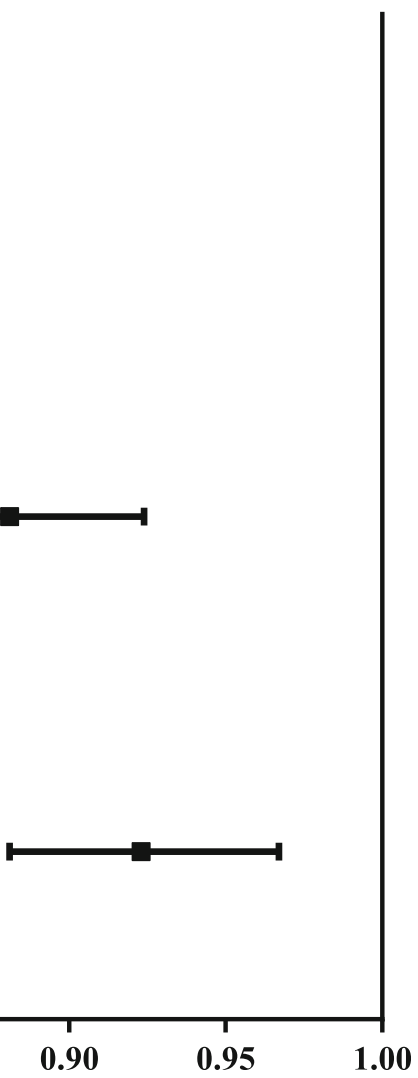

0.70

0.75

0.80

0.85

0.90

0.95

1.00

\section{Relative risks of CHD event rate}

Fig. 1 Relative Risk of CHD Event. SBP/smoking/lipid indicates that the combined effect of all three interventions was able to reduce all three risk factors. SBP/smoking indicates that the combined effect of both interventions was able to reduce both risk factors. CHD coronary heart disease, SBP systolic blood pressure 
SBP/Smoking/Lipid

$0.541(0.531-0.552)$

SBP/Smoking

$0.705(0.693-0.718)$

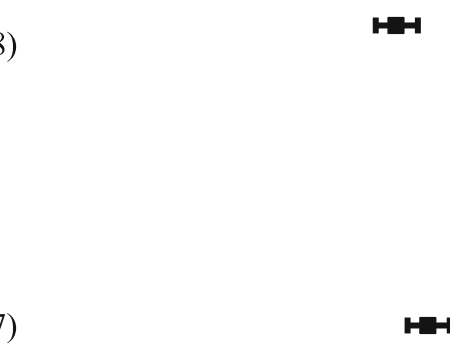

SBP

$0.724(0.712-0.737)$

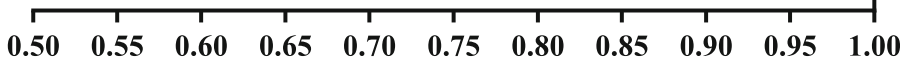

Relative risks of stroke event rate

Fig. 2 Relative Risk of Stroke Event. SBP/smoking/lipid indicates that the combined effect of all three interventions was able to reduce all three risk factors.SBP/smoking indicates that the combined effect of both interventions was able to reduce both risk factors. CHD coronary heart disease, SBP systolic blood pressure

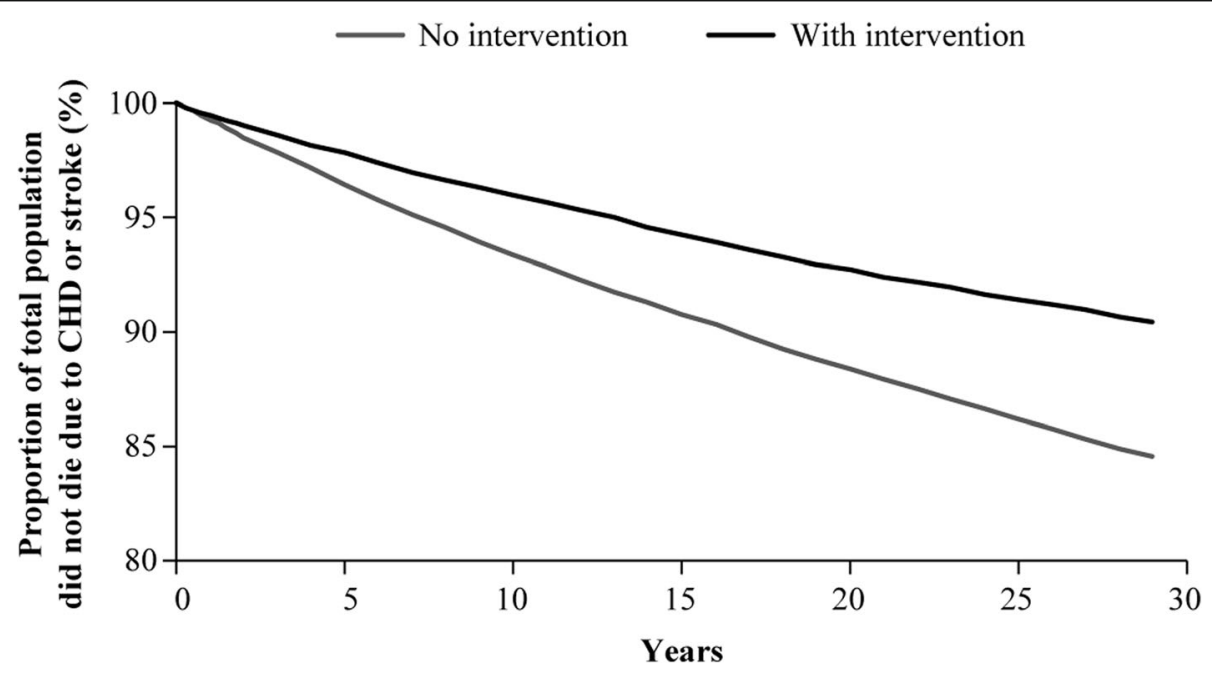

Fig. 3 Impact of SBP reduction, smoking cessation, and lipid control on mortality due to CHD or stroke. CHD coronary heart disease, SBP systolic blood pressure 
Table 7 Event rate per 100,000 people and relative risk in subgroups

\begin{tabular}{|c|c|c|c|c|c|c|c|c|c|}
\hline \multirow[t]{2}{*}{ Subgroup } & \multirow[t]{2}{*}{ Intervention } & \multicolumn{2}{|l|}{$\mathrm{CHD}$} & \multicolumn{2}{|l|}{$\underline{\text { Stroke }}$} & \multicolumn{2}{|c|}{ CHD-related death $^{\mathbf{a}}$} & \multicolumn{2}{|c|}{ Stroke-related death ${ }^{\mathbf{a}}$} \\
\hline & & Event rate & Relative risk & Event rate & Relative risk & Event rate & Relative risk & Event rate & Relative risk \\
\hline \multirow[t]{2}{*}{ Age $\geq 60$ years } & Without intervention & 404.1 & & 2568.5 & & 210.2 & & 720.2 & \\
\hline & SBP & 370.2 & 0.92 & 1892.0 & 0.74 & 195.7 & 0.94 & 536.3 & 0.75 \\
\hline \multirow[t]{2}{*}{ Age $<60$ years } & Without intervention & 150.3 & & 918.9 & & 70.2 & & 209.6 & \\
\hline & SBP & 125.6 & 0.84 & 632.8 & 0.69 & 60.0 & 0.85 & 146.4 & 0.70 \\
\hline \multirow[t]{2}{*}{ Gender, female } & Without intervention & 149.9 & & 1875.9 & & 81.0 & & 491.7 & \\
\hline & SBP & 143.9 & 0.96 & 1378.8 & 0.74 & 76.6 & 0.95 & 361.5 & 0.74 \\
\hline \multirow[t]{2}{*}{ Gender, male } & Without intervention & 455.6 & & 1679.3 & & 218.2 & & 471.9 & \\
\hline & SBP & 410.0 & 0.91 & 1172.1 & 0.70 & 193.6 & 0.89 & 332.4 & 0.71 \\
\hline \multirow[t]{2}{*}{$\mathrm{SBP} \geq 140 \mathrm{mmHg}$} & Without intervention & 340.0 & & 2393.8 & & 173.0 & & 637.6 & \\
\hline & SBP & 270.2 & 0.80 & 1331.4 & 0.56 & 137.6 & 0.81 & 351.9 & 0.56 \\
\hline \multirow[t]{2}{*}{$\mathrm{SBP}<140 \mathrm{mmHg}$} & Without intervention & 174.6 & & 800.2 & & 85.7 & & 216.8 & \\
\hline & SBP & 223.9 & 1.28 & 910.8 & 1.14 & 110.8 & 1.29 & 245.6 & 1.13 \\
\hline
\end{tabular}

CHD coronary heart disease, SBP systolic blood pressure

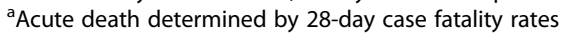

year. In China, there were 2.04 million deaths due to hypertension in 2010 [19]. The casualty is increasing over time. Applying the intervention of our CBDM study even more broadly in China could save many lives.

Given the small proportion of smokers (11\% reported from CBDM study) in the baseline population and the smoking cessation rate of $42 \%$ through the intervention program, the smoking population was too small in the study to capture significant impact on incidence of stroke.

Based on our CBDM experience, we suggest investigating further, how the effects of hypertension management differ between urban and rural areas, between different insurance schemes, or different management schemes (i.e. health management only, health management plus basic medical services model or integrated care model).

This analysis has several strengths. The level of BP control in the model simulation was based on the observation in the real-world CBDM study. In addition, based on a DES model, the analysis captured change in $\mathrm{CV}$ risk factors at the individual level and more accurately estimated the impact on CV event risk. The DES model also accounted for the elevation of future event risks when a CV (CHD or stroke) event occurs.

The impact of hypertension control on long-term clinical outcomes in our study is consistent with the previous findings. The similar results were proven in a Chinese prospective cohort study with 5006 eligible hypertensive patients aged 60 years or older during 4.8 years of followup [20]. Compared with the reference group of $\mathrm{BP}<140 /$ $90 \mathrm{mmHg}$, the risks of all-cause death, CV death, and stroke were significantly increased in the group with $\mathrm{BP}$ of $140-149 /<90 \mathrm{mmHg}$ [20]. A similar trend was also reported in the study by Stevens et al. [21].
We recognize that there are limitations to this analysis. First, the analysis was based on risk equations that were developed from older data and a different population. It is possible that the equations were not able to capture the impact of some risk factors in certain subpopulations and led to uncertainty in some of the subgroup results. In addition, the efficacy of intervention was based on 2 years of data. The effect of intervention was assumed to remain stable for the entire model time horizon. In reality, there may be a lack of adherence to the management of $\mathrm{CV}$ (CHD and stroke) risk factors not captured in the model, and treatment effect may fluctuate. Last, and most importantly, deaths due to CHD or stroke were captured only by the 28-day case fatality rates. Long-term impact of CHD and stroke on mortality was not modeled due to data limitations. Had the model captured long-term impact of CHD and stroke on mortality, the clinical benefits of the intervention are expected to be magnified. Despite the limitations, the analysis was able to provide a range of estimation that will shed light on the impact of hypertension control in the real-world practice.

\section{Conclusion}

The implementation of the CBDM study in China's Xinjiang Uygur Autonomous Region is expected to significantly reduce the incidence of $\mathrm{CV}$ events, strokes, and CV-related deaths.

\section{Abbreviations}

BMI: Body mass index; BP: Blood pressure; CBDM: Community-based disease management; CHD: Coronary heart disease; CMCS: Chinese Multi-Provincial Cohort Study; CV: Cardiovascular; CVD: Cardiovascular diseases; DES: Discreteevent simulation; HDL-C: High-density lipoprotein cholesterol; ISPOR: International Society for Pharmacoeconomics and Outcomes Research; MI: Myocardial infarction; PRC-USA: People's Republic of China - 
United States of America; SBP: Systolic blood pressure; WHO: World Health Organization

\begin{abstract}
Acknowledgments
These data were presented in part at ISPOR 19th Annual European Congress, Vienna, Austria-2016 (PCV9) and the 27th Great Wall International Congress of Cardiology, Beijing, China-2016 (GW27). The authors thank Caroline Moreau for her editorial and production assistance in the submission of this article. Authors thank Jitendriya Mishra, Novartis Healthcare Pvt. Ltd. Hyderabad, India for providing editorial assistance which encompassed manuscript revision, formatting, referencing, preparing tables and figures, incorporating the authors' revisions, finalizing, and submission all under the direction of the authors.
\end{abstract}

\section{Authors' contributions}

SP, YX, and TK worked on all aspects of the project and manuscript: conception, design, and implementation of the model; analysis and interpretation of the data; and drafting and revision of the manuscript. YL and CM contributed on the CBDM study design and data synthesis. MM, HX, $\mathrm{LL}, \mathrm{ZW} 1, \mathrm{ZW} 2$ and GY contributed in CBDM study data collection. TH, DH, and WB participated in data interpretation, and the drafting and revisions of the manuscript. ND participated in the critical review of the manuscript. All authors have read and approved the final version of this paper, and agreed to be accountable for all aspects of the work.

\section{Funding}

Novartis Pharma AG provided funding for this project.

\section{Availability of data and materials}

The datasets used and/or analyzed during the current study are available from the corresponding author on reasonable request.

\section{Ethics approval and consent to participate}

The study was approved by the ethics committees of the Peking University, School of Public Health, and the study was conducted in accordance with the International Council on Harmonization $(\mathrm{ICH}, \mathrm{E} 6)$ guidelines and Declaration of Helsinki and its subsequent revisions. The data was collected by the Xinjiang CDC and the patient consents were received written before the survey.

\section{Consent for publication}

Not applicable.

\section{Competing interests}

$Y L$ is a faculty member at Peking University. CM was a graduate student at Peking University. MM, HX, ZW1 and ZW2 are employees of Health and Family Planning Commission of Xinjiang Uygur Autonomous Region, Urumqi, China. LL and GY are employees of Xinjiang Uygur Autonomous Region Center for Disease Control and Prevention, Urumqi, China. YX, SP and TK are salaried employees of Evidera, and are not allowed to accept remuneration from any clients for their services. Evidera received funding from Novartis to conduct the study and develop this manuscript. ND is the CEO of Onom foundation, Mongolia. TH, DH, and WB are employees of Novartis Group. Authors have no other competing interests to declare.

\section{Author details}

${ }^{1}$ School of Public Health, Peking University, No. 38 Xueyuan Road, Haidian District 100191, Beijing, China. ${ }^{2}$ Health and Family Planning Commission of Xinjiang Uygur Autonomous Region, Urumqi, China, No.191 Longquan Street Tianshan Region, Urumqi 830000, Xinjiang, China. ${ }^{3}$ Xinjiang Uygur Autonomous Region Center for Disease Control and Prevention, Urumqi, China, No.380, No.1 Jianquan Street Tianshan Region, Urumqi 830000, Xinjiang, China. ${ }^{4}$ Novartis Pharmaceuticals China, No.4218 Jinke Road ZhangJiang Hi-Tech Park, Pudong 201203, Shanghai, China. ${ }^{5}$ Shanxi Province Weinan City Center for Disease Control and Prevention, Weinan, China, No.5 Zhanbei Road, Weinan City 714000, Shanxi, China. ${ }^{6}$ Evidera, Metro Building, 6th Floor, 1 Butterwick, London W6 8DL, UK. ${ }^{7}$ Sandoz International AG, Lichtstrasse 35, 4056 Basel, Switzerland. ${ }^{8}$ Onom Foundation, 3 Bogd Javzandamba 15 khoroo, Ulaanbaatar 17011, Mongolia. ${ }^{9}$ Novartis Pharma AG, Fabrikstrasse 12-1.03.14A, 4056 Basel, Switzerland.
Received: 12 October 2019 Accepted: 22 June 2020

Published online: 29 June 2020

\section{References}

1. Chen W, Gao R, Lisheng L, et al. Report on cardiovascular disease in China 2015 (in Chinese). Chin Circ J. 2016;31(6).

2. Wang W, Hu SS, Kong LZ, Gao RL, Zhu ML, Wang WY, Wu ZS, Chen WW, Yang JG, Ma LY, et al. Summary of report on cardiovascular diseases in China, 2012. Biomed Environ Sci. 2014;27(7):552-8.

3. Zhang X, Feldmam R, Wu Z, Zhang Y, Yu X, Liu L. Lb03.09: Therapeutic Inertia Is a Major Determinant of BP Control Rates in China. A Hypertension Attitude Perspectives and Needs (Happen) Study Report. J Hypertens. 2015; 33 Suppl 1:e128.

4. Caro JJ, Briggs AH, Siebert U, Kuntz KM, Force I-SMGRPT. Modeling good research practices--overview: a report of the ISPOR-SMDM modeling good research practices task Force--1. Value Health. 2012;15(6):796-803.

5. Karnon J, Stahl J, Brennan A, Caro JJ, Mar J, Moller J, Force I-SMGRPT. Modeling using discrete event simulation: a report of the ISPOR-SMDM modeling good research practices task Force--4. Value Health. 2012;15(6): 821-7.

6. Caro JJ, Möller J, Stahl J, Karnon J. Discrete event simulation for health technology assessment. New York, NY: Chapman and Hall/CRC; 2015.

7. Liu J, Hong Y, D’Agostino RB Sr, Wu Z, Wang W, Sun J, Wilson PW, Kannel WB, Zhao D. Predictive value for the Chinese population of the Framingham CHD risk assessment tool compared with the Chinese multiprovincial cohort study. JAMA. 2004;291(21):2591-9.

8. Wu Y, Liu X, Li X, Li Y, Zhao L, Chen Z, Li Y, Rao X, Zhou B, Detrano R, et al. Estimation of 10-year risk of fatal and nonfatal ischemic cardiovascular diseases in Chinese adults. Circulation. 2006;114(21):2217-25.

9. Moran A, Gu D, Zhao D, Coxson P, Wang YC, Chen CS, Liu J, Cheng J, BibbinsDomingo K, Shen YM, et al. Future cardiovascular disease in China: markov model and risk factor scenario projections from the coronary heart disease policy model-China. Circ Cardiovasc Qual Outcomes. 2010;3(3):243-52.

10. WHO - Life tables by country: China 2013 [http://apps.who.int/gho/data/ view.main.60340]

11. China Demographics Profile 2014 [http://www.indexmundi.com/china/ demographics_profile.html].

12. Universal health coverage [http://www.who.int/universal_health_coverage/ en/ (Assessed on 28 August 2019)].

13. The World Health Report [http://www.who.int/whr/2008/08_contents_en.pdf].

14. Cene CW, Roter D, Carson KA, Miller ER 3rd, Cooper LA. The effect of patient race and blood pressure control on patient-physician communication. J Gen Intern Med. 2009;24(9):1057-64.

15. Wong MC, Wang HH, Wong SY, Wei X, Yang N, Zhang Z, Li H, Gao Y, Li DK, Tang J, et al. Performance comparison among the major healthcare financing systems in six cities of the Pearl River Delta region, mainland China. PLoS One. 2012;7(9):e46309.

16. Ma YT, Huang Y, Liu F, Yang Y-N, Li X-M, Sun M-H, Juan. General Cardiology: Hypertension, Prevention and Lipids Epidemiological Survey of Hypertension in General Adult Population of Different Nationalities in Xinjiang. J Am Coll Cardiol. 2011;57(14).

17. 2016 Chinese guideline for the management of dyslipidemia in adults [https://www.ncbi.nlm.nih.gov/pubmed/27903370].

18. Yuan YYNYTMWWJWLL. Summary of "2015 China adult tobacco survey". Chin J Health Manag. 2016;10(02):85-7.

19. Li GX, Zhou B, Qi GX, Zhang B, Jiang DM, Wu GM, Ma B, Zhang P, Zhao QR, $\mathrm{Li}$ J, et al. Current trends for ST-segment elevation myocardial infarction during the past 5 years in rural areas of China's Liaoning Province: a multicenter study. Chin Med J. 2017;130(7):757-66.

20. Zheng L, Li J, Sun Z, Zhang X, Hu D, Sun Y. Relationship of blood pressure with mortality and cardiovascular events among hypertensive patients aged $>/=60$ years in rural areas of China: a strobe-compliant study. Medicine (Baltimore). 2015;94(39):e1551.

21. Stevens W, Peneva D, Li JZ, Liu LZ, Liu G, Gao R, Lakdawalla DN. Estimating the future burden of cardiovascular disease and the value of lipid and blood pressure control therapies in China. BMC Health Serv Res. 2016;16: 175.

\section{Publisher's Note}

Springer Nature remains neutral with regard to jurisdictional claims in published maps and institutional affiliations. 